\title{
Niveles de EGF y VEGF en el plasma rico en plaquetas antes y después de antiagregantes plaquetarios
}

\author{
EGF y VEGF levels in platelet-rich plasma before and after antiplatelet drugs
}

Título corto en español: EGF y VEGF en el plasma rico en plaquetas y los antiagregantes

\author{
- Maczy González', Melvis Arteaga-Vizcaíno², Ana Ruiz³, Olga Briceño ${ }^{3}$, Maribel Quintero4, Jesús Estévez², José Urdaneta ${ }^{5}$ \\ Jefa de la Cátedra de Hematología, Escuela de Bioanálisis, Facultad de Medicina, LUZ. \\ Instituto de Investigaciones Clínicas, Facultad de Medicina. \\ Catedra de Hematicina. \\ Sátedra de Anatomía, Escuela de Bioanálisis, Facultad de Medicina, Universidad del Zulia (LUZ).
}

\section{Resumen}

Introducción: el plasma rico en plaquetas (PRP) es un producto autólogo rico en factores de crecimiento indispensables en la regeneración tisular.

Objetivo: cuantificar las concentraciones de VEGF y EGF en el PRP antes y después de los antiagregantes plaquetarios: aspirina (AAS) y clopidogrel; y su correlación con el recuento plaquetario.

Material y método: se estudiaron en 32 sujetos sanos concentraciones de EGF y VEGF (Elisa) en PRP, plasma pobre en plaquetas (PPP), exudado y lisado, antes y después de aspirina (AAS) y clopidogrel.

Resultados: la concentración de EGF en el PRP y sus subproductos antes y después del tratamiento con AAS o clopidogrel no mostró diferencia significativa para ninguno de los derivados plaquetarios analizados; no así para el VEGF, donde se encontró diferencia significativa en el subproducto 'lisado' $(p<0,05)$. Se apreció correlación entre el recuento plaquetario en el PRP basal $\left(578,25 \pm 125,33 \times 103 \times \mathrm{mm}^{3}\right)$ frente a la concentración basal de EGF $(296 \pm 203,6 \mathrm{pg} / \mathrm{ml})(\mathrm{r}=0,726, p<0,01)$, en el grupo tratado con AAS, pero no para el clopidogrel.

Conclusiones: las cifras plaquetarias, en los subproductos de concentrados plaquetarios o sangre periférica, no son predictivas de las concentraciones de FC, dado que otros elementos pueden influenciar la liberación final de estos. De igual manera, los datos obtenidos parecen apuntar la utilidad clínica que puede tener la aplicación de PRP en sujetos tratados con AAS o clopidogrel en el momento que así lo ameriten.

Palabras clave: PRP, EGF, VEGF, AAS, clopidogrel.

\begin{abstract}
Background: The platelet-rich plasma (PRP) is considered rich product autologous growth factors essential in tissue regeneration. Objective: to quantify the concentrations of VEGF and EGF in the PRP before and after platelet aggregation: aspirin (ASA) and clopidogrel; and its correlation with the platelet count.

Material and methods: we studied in 32 healthy subjects, concentrations of EGF and VEGF (Elisa) in PRP, platelet-poor (PPP), exudate, lysate before and after of aspirin (AAS) and clopidogrel (CLO).

Results: the concentration of EGF in the PRP and its products before and after treatment with AAS or clopidogrel, showed no significant difference for none of the analyzed platelet derivatives; not so for the VEGF where significant difference was found in the 'lysate' by-product $(p<0.05)$. Appreciated correlation between platelet count in the basal PRP from healthy subjects $\left(578.25 \pm 125.33 \times 103 \times \mathrm{mm}^{3}\right)$ versus the baseline concentration of EGF in the group treated with ASA $(296 \pm 203.6 \mathrm{pg} / \mathrm{ml})(r=0.726, p<0.01)$, but not for clopidogrel. Conclusions: platelet numbers, both by-products of concentrated platelet or peripheral blood, no are predictive of the concentrations of FCs, since other elements can influence the final release of them. Similarly the data seem to point the clinical utility that can take the implementation of PRP in subjects treated with ASA or clopidogrel, at the time that needs you so.
\end{abstract}

Key words: PRP, EGF, VEGF, aspirin, clopidogrel.

Fuente de financiación: Consejo de Desarrollo Científico, Humanístico y Tecnológico (Condes)

Conflictos de interés:

Derechos de reproducción: ๑ 2016 González et al. Este es un artículo de acceso libre distribuido bajo los términos de la licencia Creative Commons BY-NC-ND, la cual otorga permiso público para compartir y usar los artículos bajo la condición de atribución al autor, no modificación de su contenido y no uso con fines comerciales.

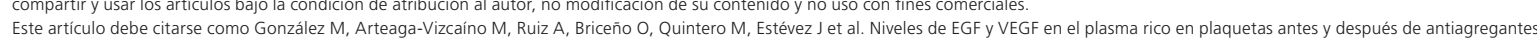
plaquetarios. Revista Colombiana de Hematología y Oncología. 2016;3(2):24-31.

\section{DATOS DE CONTACTO}

Correspondencia: Maczy González Rincón. Cátedra de Hematología, Escuela de Bioanálisis, Facultad de Medicina, Universidad del Zulia (Maracaibo, Estado Zulia, Venezuela). Final Avenida 20 al lado de la Maternidad Dr. Castillo Plaza. Teléfonos: 0261-412 7225 - 0424-669 2707.

Correos electrónicos: maczygonzalez@hotmail.com, maczy.gonzalez@gmail.com 


\section{Introducción}

Las plaquetas cumplen un papel protector del organismo cuando ocurre un traumatismo vascular, no solo porque intervienen en la hemostasia primaria formando el coágulo plaquetario, sino también por el rol que juegan en la regeneración tisular debido a su alta concentración en factores de crecimiento (FC). Los FC son polipéptidos producidos y secretados por la mayoría de las células del organismo en respuesta a un estímulo específico, pero la rapidez con la cual las plaquetas se acumulan en los sitios de injuria las hace ser la fuente más relevante. Entre estos factores están: el vásculo-endotelial (VEGF), epidermal (EGF), derivado de las plaquetas (PDGF), transformante beta (TGF $\beta$ ) y el similar a la insulina (IGF) $)^{1,2}$. De allí que el plasma rico en plaquetas (PRP) se reconoce como un poderoso agente hemostático-adhesivo ${ }^{3}$ y una fuente potente de estos factores ${ }^{4-9}$.

El PRP es una fracción del plasma que tiene una concentración de plaquetas, su número oscila entre 2 a 5 veces al reportado en sangre periférica. Este concentrado plaquetario se obtiene generalmente por centrifugación de la sangre, pudiéndose conseguir en forma líquida o en gel. Este último se logra al activar las plaquetas con trombina o calcio, resultando en un producto de consistencia gelatinosa que contiene fibrina además de moléculas de adhesión celular (fibronectina y vitronectina) y FC, los cuales participan en el proceso de la coagulación, cicatrización y regeneración tisular $^{4-7}$. El PRP, al ser aplicado en forma tópica sobre una herida, potencia los mecanismos de regeneración, de manera rápida y eficaz, sin efectos secundarios, pues es obtenido del propio paciente ${ }^{8,9}$.

Hoy día, los FC, como el EGF, VEGF y PDGF, son obtenidos a través de bioingeniería genética, y se emplean en la cicatrización de quemaduras, úlceras, aplicaciones pospeeling, entre otras situaciones médicas ${ }^{10}$.

El VEGF, también denominado factor de permeabilidad vascular (VPF), es el más poderoso de los promotores del crecimiento vascular conocidos, y ejerce un rol directo en el control del comportamiento de las células endoteliales, tal como proliferación, migración, especialización y sobrevivencia11-16. Por su parte, el EGF o factor de crecimiento epidermal posee funciones mitogénica, proapoptótica, de migración y diferenciación de células epiteliales, fibroblastos, células renales y gliales a partir de células mesenquimales; también estimula la proliferación y diferenciación de la epidermis, dermis, epitelio corneal, pulmones y de tráquea durante la reparación tisular ${ }^{17-18}$.

La aplicación de PRP en pacientes atendidos en diferentes áreas médicas ha demostrado ser de gran utilidad por su gran influencia en la regeneración tisular; no obstante, aquellos sujetos tratados con medicamentos que alteran la función plaquetaria no se consideran elegibles para la aplicación de PRP, entre ellos están los que padecen enfermedades cardiovasculares, quienes reciben antiagregantes plaquetarios, como el ácido acetilsalicílico (AAS) (aspirina) o tienopiridinas (clopidogrel/ ticlopidina $)^{19-22}$.

EI AAS inhibe de manera irreversible la enzima ciclooxigenasa (COX-1) mediante acetilación en un residuo de serina del sitio activo de la COX de las plaquetas, interrumpiendo la transformación de ácido araquidónico en tromboxano $\mathrm{A} 2\left(\mathrm{TXA}_{2}\right)$, que tiene un potente efecto sobre la agregación plaquetaria. Se administra por vía oral y se absorbe rápidamente por el tracto digestivo. Es hidrolizada parcialmente a ácido salićlico durante su paso a través del hígado y se distribuye ampliamente por todos los tejidos del organismo. Después de la ingestión y dependiendo de las dosis administradas, se encuentran salicilatos en plasma a los 5-30 minutos y las concentraciones máximas se obtienen a los 0,25-2 horas; su efecto antitrombótico se observa con intervalos de 24 a 48 horas. A dosis bajas (30 a $325 \mathrm{mg}$ ) se produce el efecto antiagregante y a dosis mayores (500-1.300 mg/día) se condiciona su efecto analgésico y antipirético ${ }^{23}$.

Por su parte, el clopidogrel inhibe la agregación plaquetaria por su acción sobre los receptores plaquetarios $\left(\mathrm{P}_{2} \mathrm{Y}_{12}\right)^{24}$. Se administra por vía oral, su absorción es del $50 \%$ y no es afectada significativamente por los alimentos. Las concentraciones plasmáticas máximas se consiguen una hora después de la dosis de $75 \mathrm{mg}$ y dos horas después ya no son detectables en plasma. El clopidogrel y su principal metabolito se unen de forma reversible a las proteínas plasmáticas (98 y 94\%, respectivamente). Luego de una dosis única, la agregación plaquetaria inducida por el ADP se ve inhibida de forma dependiente de la dosis en voluntarios sanos. Con dosis repetidas de 50 a $100 \mathrm{mg}$ por día, la inhibición de la agregación plaquetaria suele iniciarse al segundo día, alcanzando su máximo nivel a los 3-7 días, y es inhibida en un $40-60 \%{ }^{25}$. 
Tanto el AAS como el clopidogrel se utilizan para prevenir episodios de trombosis en pacientes con enfermedad vascular periférica, infarto de miocardio, ictus, accidente cerebrovascular, cardio o perivascular, en los cuales las plaquetas son el principal elemento en la formación de un trombo; se busca pues inducir un efecto inhibitorio sobre la agregación plaquetaria, pero esto podría a su vez interferir en la liberación de los FC contenidos en sus gránulos $\alpha^{23,24}$.

Se reporta aumento de la morbimortalidad en pacientes con las patologías antes señaladas, que cursan además con lesiones de tejidos (espontáneas o provocadas), por lo que se hace necesario acelerar la reparación tisular. Para ello se han empleado diferentes terapias que permitan la mejoría y curación del paciente, como se describe en áreas como medicina interna, traumatología, odontología, cirugía plástica y oftalmología. El objetivo de estas terapias es acelerar la curación de esas lesiones e incorporar al paciente a sus actividades cotidianas en el menor tiempo posible, como se reporta en la aplicación de PRP autólogo en forma tópica, líquida o en gel, en pacientes con DM con úlceras en miembros inferiores ${ }^{26-28}$.

Conociendo el mecanismo de acción y efecto terapéutico de una droga antiagregante plaquetaria en diversas patologías médicas y el empleo del PRP para acelerar la regeneración y reparación tisular, este trabajo se propone determinar las concentraciones de EGF y VEGF en el PRP y sus subproductos en sujetos sanos tratados con antiagregantes plaquetarios y su correlación con el recuento plaquetario.

\section{Material y métodos}

El diseño del presente estudio es de campo, el tipo es experimental y longitudinal ${ }^{29}$.

La población objeto de estudio estuvo conformada por todos los sujetos adultos, de ambos sexos, aparentemente sanos, que acudieron al Instituto de Investigaciones Clínicas Dr. Américo Negrette de la Facultad de Medicina de la Universidad del Zulia. Esta población es de carácter finita, es decir, menos de 100 mil sujetos $^{29}$.

La muestra se calculó de manera no aleatoria e intencionada ${ }^{29}$ y correspondió a 32 sujetos. Los criterios de selección que se tomaron en cuenta fueron los siguientes:

\section{Inclusión}

- Edad entre 18 y 50 años.

- En ayunas.

- Sin enfermedad clínica de base conocida.

- Aparentemente sanos.

- Resultados normales en el estudio de agregación plaquetaria que se realice antes de la ingestión de las drogas antiplaquetarias que se utilizaron en este análisis.

\section{Exclusión}

- Haber ingerido antiagregantes plaquetarios 11 días antes del estudio.

Para disminuir el sesgo de la muestra, en el muestreo la técnica que se utilizó correspondió a los 32 primeros sujetos que cumplieron con todos los criterios de inclusión y que deseaban participar en este estudio.

Todos los sujetos fueron sometidos a un examen clínico y de laboratorio exhaustivo, con el fin de descartar enfermedades sistémicas. Estos se distribuyeron de la siguiente manera:

- Grupo A: 32 sujetos sin tratamiento previo con drogas antiagregantes plaquetarias. Estos mismos conformaron el grupo $B$.

- Grupo B: se dividió a su vez en dos subgrupos:

- B1: constituidos por 16 sujetos que recibieron aspirina a una dosis de $100 \mathrm{mg}$ una sola por un día.

- B2: 16 individuos que recibieron clopidogrel a una dosis de $75 \mathrm{mg}$ en dosis única un solo día.

\section{Técnicas de recolección de las muestras}

Tanto a los sujetos del grupo A como B, a las 24 horas del consumo del fármaco respectivo, en ayunas y previa asepsia de la zona, se les extrajo $16 \mathrm{ml}$ de sangre venosa antecubital; para ello se utilizaron mariposas $\mathrm{N}^{\circ}$ 21, empleando la técnica de la doble jeringa para evitar la activación de las plaquetas.

1. La primera jeringa contenía $2,5 \mathrm{ml}$ de sangre que fue dispensada en tubos de vidrio con EDTA para estudio de hematología, recogiendo los datos concernientes a plaquetas y glóbulos blancos, empleando un contador automático de células Beckman Coulter AC-T. Se consideraron valores normales para plaquetas entre 150.000 a $450.000 \mathrm{x} \mathrm{mm}^{3}$ en sangre periférica $^{30}$. 
2. La segunda jeringa contenía $13,5 \mathrm{ml}$ de sangre venosa, que se distribuyó como se describe a continuación:

a) $4,5 \mathrm{ml}$ se dispensaron en un tubo plástico que contenía $0,5 \mathrm{ml}$ de citrato de sodio al $3,8 \%$ y se centrifugó a 800 rpm (180 g) durante 10 minutos, a temperatura ambiente, para obtener PRP; luego el remanente de cada muestra se centrifugó a 4.500 rpm durante 20 minutos para obtener plasma pobre en plaquetas (PPP). Se realizó agregación plaquetaria según el método turbidimétrico de Born ${ }^{31}$, empleando un agregómetro Chrono-log (Corp. Haverton, PA, USA). El resultado obtenido se expresó en porcentaje.

b) $9 \mathrm{ml}$ se colocaron en otro tubo plástico que contenía $1 \mathrm{ml}$ de citrato de sodio al 3,8\% y fueron sometidos a centrifugación, en centrífuga clínica a una velocidad de 1.400 rpm por 7 minutos, a $267 \mathrm{G}$ para la obtención de PRP; del volumen total del PRP obtenido se extrajeron $0,5 \mathrm{ml}$ que correspondieron a PPP, siguiendo la técnica de Anitua ${ }^{32}$. El resto se tomó con un pipeteado muy meticuloso y con una punta distinta, hasta la zona que se encuentra por encima de la fracción roja, representando el PRP; $1 \mathrm{ml}$ de este PRP se alicuotó para recuento de plaquetas y medición de FC. A otro $\mathrm{ml}$ del PRP se le adicionó 50 ul de cloruro de calcio al 10\% $\left(\mathrm{CaCl}_{2}\right)$ que se mezcló y se dejó reposar por 15-30 minutos para obtener un gel de PRP. Luego este se centrifugó a 2.500-3.000 rpm por 10 minutos, para obtener exudado (Ex). Otro ml del PRP sin activar se sometió a segunda centrifugación a 3.000 rpm x 10 minutos, se obtuvo un concentrado plaquetario que se sometió a un proceso repetido de congelación $\left(-70^{\circ} \mathrm{C}\right)$ y descongelación $\left(37^{\circ} \mathrm{C}\right)$, por cinco veces, y se obtuvo el producto que denominamos lisado plaquetario.

El PPP, PRP sin activar, el exudado del PRP gelificado y el lisado se distribuyeron en alícuotas y se almacenaron en tubos plásticos Eppendorf $\mathrm{a}-70^{\circ} \mathrm{C}$ en un ultracongelador vertical (Forma Scientific U95-18), lugar donde se conservaron hasta el análisis del FC, empleando Elisa ${ }^{33}$.

\section{Método para la determinación de factores de crecimiento}

Las concentraciones del factor de crecimiento EGF y VEGF se midieron en muestras y estándares, empleando el método de Elisa, cuyos kits fueron suministrados por
Abcam (Abcam Inc., Cambridge, USA: 1 Kendall Square, Ste B2304 Cambridge, MA 02139-1517, USA) con números de lote: GR 85403-1, GR85404-1 y GR 56644-1, y fueron ejecutados de acuerdo con las instrucciones del manufacturador.

\section{Análisis de los resultados}

Los datos se muestran en tablas en valores absolutos y porcentajes, así como media +/- 1 desviación estándar. Para la comparación de las variables en estudio, se utilizó la prueba $t$ de student, y, para el estudio de correlación, se aplicó la prueba de Pearson, se consideró $\mathrm{p}<0,05$ como la menor probabilidad estadística ${ }^{34}$.

\section{Aspectos éticos}

A todos los sujetos se les requirió consentimiento informado por escrito antes de ser incluidos en el estudio, y se les identificó a través de números; se contó, así mismo, con la aprobación del Comité de Ética de la institución (Instituto de Investigaciones Clínicas, Facultad de Medicina, LUZ) ${ }^{35}$ y se procedió conforme con los principios de la Declaración de Helsinki de $1975^{36}$ (actualizada en 2013) y las recomendaciones elaboradas por el Consejo de Organizaciones Internacionales de las Ciencias Médicas (CIOMS, por su sigla en inglés) en $2002^{37}$.

\section{Resultados}

La tabla 1 muestra las concentraciones de EGF en el PRP y sus respectivos subproductos de sujetos sanos antes y después del tratamiento con AAS/clopidogrel. Tanto en el grupo tratado con AAS como el de clopidogrel los niveles de este FC no mostraron diferencias estadísticamente significativas cuando se compararon antes y después de la ingestión de estos medicamentos, para ninguno de los diferentes derivados plaquetarios analizados.

Los valores del VEGF se presentan en la tabla 2. Se encontró que los niveles plasmáticos de este factor luego del tratamiento con AAS solo presentaron diferencia estadísticamente significativa para el subproducto 'lisado' $(p<0,05)$. Mientras que para el grupo tratado con clopidogrel, se observó diferencia estadísticamente significativa en relación con el lisado $(p<0,05)$ y se notó que los valores más altos se encontraron en el PRP y lisado, tanto antes como después del tratamiento. 
Tabla 1. concentración de EGF (pg/ml) en el PRP y sus subproductos de sujetos sanos, antes y después del tratamiento con aspirina (AAS) y clopidogrel

\begin{tabular}{|c|c|c|c|c|c|c|}
\hline Subproducto & Basal & AAS & $\mathbf{p}$ & Basal & Clopidogrel & $\mathbf{p}$ \\
\hline $\begin{array}{c}\text { PPP } \\
n=16\end{array}$ & $96 \pm 141,1$ & $172,75 \pm 167,4$ & 0,1711 & $11,9 \pm 6,8$ & $8,25 \pm 2,75$ & 0,0557 \\
\hline $\begin{array}{c}\text { PRP } \\
n=16\end{array}$ & $296,1 \pm 203,6$ & $353,95 \pm 204,5$ & 0,4289 & $51,75 \pm 14,35$ & $58,75 \pm 29,15$ & 0,3956 \\
\hline $\begin{array}{c}\text { Exudado } \\
n=16\end{array}$ & $150,25 \pm 120,8$ & $159,7 \pm 71,1$ & 0,7893 & $29,75 \pm 12,95$ & $36,8 \pm 11,95$ & 0,1200 \\
\hline $\begin{array}{l}\text { Lisado } \\
n=16\end{array}$ & $280 \pm 70$ & $275 \pm 35$ & 0,8000 & $37 \pm 18,9$ & $48 \pm 13$ & 0,0647 \\
\hline
\end{tabular}

Tabla 2. concentración de VEGF (pg/ml) en el PRP y sus subproductos de sujetos sanos, antes y después del tratamiento con aspirina (AAS) y clopidogrel

\begin{tabular}{|c|c|c|c|c|c|c|}
\hline Subproducto & Basal & AAS & $\mathbf{p}$ & Basal & Clopidogrel & $\mathbf{p}$ \\
\hline $\begin{array}{c}\text { PPP } \\
n=16\end{array}$ & $658,8 \pm 262,68$ & $708,8 \pm 353,88$ & 0,6532 & $504 \pm 288,52$ & $542 \pm 272,12$ & 0,7042 \\
\hline $\begin{array}{c}\text { PRP } \\
n=16\end{array}$ & $833,6 \pm 446,4$ & $913,6 \pm 380,84$ & 0,5895 & $663,2 \pm 419,2$ & $680,4 \pm 383,6$ & 0.9044 \\
\hline $\begin{array}{c}\text { Exudado } \\
n=16\end{array}$ & $756,4 \pm 375,56$ & $870 \pm 387,76$ & 0,4066 & $568 \pm 366,24$ & $568,4 \pm 309,32$ & 0.9974 \\
\hline $\begin{array}{l}\text { Lisado } \\
n=16\end{array}$ & $632,8 \pm 212$ & $801,6 \pm 151,72^{\mathrm{a}}$ & 0,0147 & $728,8 \pm 387,88$ & $1076,8 \pm 534^{a}$ & 0,04343 \\
\hline
\end{tabular}

Tabla 3. Correlación del recuento plaquetario en PRP de los sujetos sanos estudiados con los niveles de EGF antes del tratamiento

\begin{tabular}{|c|c|c|}
\hline $\begin{array}{c}\text { Plaquetas } \\
\mathbf{1 0}^{\mathbf{3}} \mathbf{x} \mathbf{~ m m}^{\mathbf{3}} \text { en PRP } \\
\text { (Basal) grupo AAS }\end{array}$ & $\begin{array}{c}\text { Niveles } \\
\text { promedio y DS } \\
\text { EGF (basal) grupo } \\
\text { AAS (pg/ml) }\end{array}$ & $\begin{array}{c}\text { Correlación } \\
\text { (r) }\end{array}$ \\
\hline $578,25 \pm 125,33$ & $296 \pm 203,6$ & $0,726^{a}$ \\
\hline
\end{tabular}

En la tabla 3, se aprecia el estudio de correlación entre el recuento plaquetario en el PRP basal de sujetos sanos $\left(578,25 \pm 125,33 \times 10^{3} \times \mathrm{mm}^{3}\right)$ frente a los niveles basales promedio de EGF en el grupo del AAS $(296 \pm 203,6 \mathrm{pg} / \mathrm{ml})$ analizados en este estudio. Observándose que se pudo establecer correlación entre ambos parámetros $(r=0,726, p<0,01)$. No así para el clopidogrel.

\section{Discusión}

De los FC presentes en el PRP, el PDGF (en sus diferentes isoformas), EGF y VEGF se han constituido en los más estudiados, debido principalmente al efecto determinante en la estimulación de la regeneración tisular. Su secreción durante la agregación plaquetaria y coagulación puede llegar a estimular la quimiotaxis y la multiplicación celular, de allí su rol protagónico en el proceso de regeneración tisular y cicatrización ${ }^{38-41}$.

Al considerar los niveles de EGF antes y después del tratamiento tanto con AAS como con clopidogrel, se observa que no se encontraron diferencias significativas en ninguno de los derivados plaquetarios. Los valores basales de este FC fueron similares a los citados por otros autores ${ }^{42,43}$. Al respecto, Eppley y colaboradores (2004) determinaron el número de plaquetas y FC como el EGF en el PRP y sangre completa de 10 sujetos sanos que se someterían a cirugía plástica y se halló un incremento de $129 \pm 61 \mathrm{pg} / \mathrm{ml}$ a $470 \pm 320 \mathrm{pg} / \mathrm{ml}$ (sangre completa y PRP, respectivamente), siendo estos niveles aproximados a los valores basales y postratamiento para el grupo del AAS, no así para el grupo del clopidogrel.

Para el grupo clopidogrel, las concentraciones lucieron más bajas que las del AAS, por lo que se indica que existe una alta variabilidad individual en la producción celular, almacenamiento y secreción de los FC por parte de las plaquetas y de otras células, y que cada individuo necesitaría diferentes concentraciones de estas células, en especial de las plaquetas, para lograr una secreción de FC con efecto biológico comparable ${ }^{42-44}$.

Cuando se observan las concentraciones promedio del EGF en los grupos postratamiento con AAS y clopidogrel, se nota que los valores de este FC son más altos en el PRP con respecto al resto de los derivados plaquetarios, en el PPP son los más bajos y hay similitud entre las concentraciones arrojadas para el PRP y lisado, sin diferencias significativas para ningún derivado plaquetario. Este hallazgo no permite establecer diferencia relevante entre estos subproductos plaquetarios, pero sí confirmar lo asegurado por Woo y colaboradores (2013), quienes afirman que no existe influencia importante de la trombina o cloruro de calcio sobre la 
activación del PRP y la secreción de los FC, así como en la cantidad de plaquetas concentradas en el PRP, y, por lo tanto, la adición de estas sustancias resulta innecesaria para lograr su efectiva preparación ${ }^{43}$.

Por otro lado, se puede apreciar que los niveles postratamiento de EGF en el grupo del AAS son más elevados que los del grupo del clopidogrel, esto podría estar revelando un mayor grado de afectación de los niveles de este FC en los sujetos tratados con este último, a consecuencia posiblemente del mecanismo farmacológico diferencial que exhiben ambos fármacos ${ }^{23}$.

Al observar las concentraciones promedio del VEGF en el PRP y sus subproductos plaquetarios antes y después de la administración de AAS/clopidogrel, se puede apreciar que los valores basales coinciden con los reportados por otros investigadores ${ }^{19,21,45}$. Al respecto, Agren y colaboradores (2013) determinaron, entre otros FC, el VEGF en PRF en 10 donantes voluntarios; el PRF se obtuvo a través de un sistema comercial denominado Vivostat $\AA$. Los niveles de VEGF estuvieron en $406 \pm 141 \mathrm{pg} / \mathrm{ml}$; así mismo, reportan cierta variabilidad en la concentración de los FC medidos, indicando que esto es debido a las diferencias individuales que se pueden presentar entre donantes ${ }^{19}$. También Barsotti y colaboradores (2013) midieron el efecto del lisado plaquetario en las diferentes fases del proceso de cicatrización de heridas, obteniendo valores promedio de VEGF de $740 \pm 110 \mathrm{pg} / \mathrm{ml}$, similares a los de esta investigación ${ }^{22}$.

Cuando se aprecian los niveles promedio de VEGF en los grupos de estudio de acuerdo con el fármaco empleado, se nota que en el grupo del AAS los valores promedio más altos se encuentran en el PRP (antes y después), mientras que en el grupo del clopidogrel están en el lisado con diferencia estadísticamente significativa, tanto para el grupo del AAS como para el clopidogrel. Esto permite confirmar lo asegurado por otros autores, como Weibrich y colaboradores (2002), Perseghin y colaboradores (2005) y Barsotti y colaboradores (2013), quienes destacan que el congelamiento a muy bajas temperaturas $\left(-70^{\circ} \mathrm{C}\right.$ ) y su rápida descongelación $\left(37^{\circ} \mathrm{C}\right)$ es un método común y efectivo para lograr la liberación de los FC, sin afectación de sus concentraciones, ni de los niveles biológicamente activos ${ }^{21,22,44}$, gracias en gran medida a la lisis masiva de las plaquetas presentes en el derivado plaquetario. Este aspecto es de utilidad clínica a la hora de considerar la utilización de concentrados de PRP congelados de pacientes que reciban antiagregantes plaquetarios, como los empleados en el presente estudio.

Conjuntamente, se puede apreciar que en ambos grupos de estudio (AAS/clopidogrel) se experimentó un incremento de los valores promedio de VEGF luego del tratamiento respectivo, el cual se hace más evidente en el grupo del AAS. Esto parecería estar indicando alteración de los FC que parecen ser específicos para cada fármaco.

Es significativo resaltar que en la literatura revisada no se encontraron trabajos científicos que hubiesen llevado a cabo las determinaciones de EGF y VEGF posterior a los tratamientos con los antiagregantes plaquetarios aquí empleados, por lo cual no se pudo establecer comparación alguna con otra experiencia similar.

Al realizar los estudios de correlación entre el recuento plaquetario en el PRP y PPP de cada grupo de estudio con los niveles pre y postratamiento de cada FC, se pudo constatar que solo se estableció una correlación significativa para el conteo plaquetario del PRP basal frente a los valores pretratamiento de EGF en el PRP del grupo del AAS ( $r: 0,726)$, siendo estadísticamente significativa $(p<0,01)$, lo cual permite deducir que solo para esta relación una alteración de las cifras plaquetarias estaría directamente relacionada (o condicionaría) con un aumento o disminución de los niveles basales de EGF en el PRP de sujetos sanos, antes de ser sometidos a tratamiento con AAS.

En el mismo sentido, para el VEGF no se encontró correlación en lo que respecta a las cifras plaquetarias del PRP/PPP para cada grupo de estudio. Estos resultados coinciden con los reportados por varios autores ${ }^{42,45}$; Zimmermann y colaboradores (2001) no encontraron correlación entre el conteo de plaquetas en concentrados plaquetarios obtenidos de 15 donantes voluntarios y la concentración de los $\mathrm{FC}^{46}$.

Los resultados aquí presentados revelan que las cifras plaquetarias tanto en los concentrados o sangre periférica no son predictivas de los FC, en virtud de que existen otros factores que pueden influenciar su liberación final, como son: la activación plaquetaria y la contaminación con leucocitos ${ }^{46}$. Sin embargo, para considerar a profundidad una correlación más concluyente, se necesitaría diseñar nuevos experimentos que permitiesen comparar el recuento plaquetario y 
los niveles de FC, analizados a partir de diversos PRP provenientes de varios protocolos de centrifugación diferencial.

De igual manera, los datos obtenidos parecen tener utilidad clínica en cuanto a la aplicación de PRP en pacientes tratados con antiagregantes plaquetarios como AAS o clopidogrel en cualquier momento que así lo ameriten.

\section{Agradecimiento}

Esta investigación fue financiada por el Consejo de Desarrollo Científico, Humanístico y Tecnológico de la Universidad del Zulia (Condes), a través del proyecto VAC-CONDES-CC-0019-12. Los autores agradecen la dotación de materiales reactivos y equipo necesario para la ejecución de este trabajo de investigación. Así mismo, se contó con la colaboración del Laboratorio de Hematología del Instituto de Investigaciones Clínicas Dr. Américo Negrette de la Facultad de Medicina de la Universidad del Zulia, en cuyos laboratorios se llevó a cabo el análisis de las muestras recolectadas.

\section{Referencias}

1. Dohan DM, Choukroun J, Diss A, Dohan SL, Dohan AJ, Mouhyi J, et al. Platelet-rich fibrin (PRF): a second-generation platelet concentrate. Part II: platelet-related biologic features. Oral Surg Oral Med Oral Pathol Oral Radiol Endod. 2006;101(3):e45-50.

2. Dohan DM, Choukroun J, Diss J, Dohan SL, Dohan AJ, Mouhyi J, et al. Platelet-rich fibrin (PRF): a second-generation platelet concentrate. Part III: leucocyte activation: a new feature for platelet concentrates? Oral Surg Oral Med Oral Pathol Oral Radiol Endod. 2006;101(3):e51-5.

3. Ogino $Y$, Ayukawa $Y$, Kukita $T$, Koyano K. The contribution of platelet-derived growth factor, transforming growth factor- $\beta 1$, and insulin-like growth factor-I in platelet-rich plasma to the proliferation of osteoblast-like cells. Oral Surg Oral Med Oral Pathol Oral Radiol Endod. 2006;101(6):724-9.

4. Foster TE, Puskas BL, Mandelbaum BR, Gerhardt MB, Rodeo SA Platelet-rich plasma: from basic science to clinical applications. Am J Sport Med. 2009;37(11):2259-72.

5. Anitua $E$, Sánchez $M$, Prado $R$, Orive $G$. Plasma rich in growth factors: the pioneering autologous technology for tissue regeneration. J Biomed Mat Res A. 2011;97(4):536.

6. Sánchez AR, Sheridan PJ, Kupp LI. Is platelet-rich plasma the enhancement factor? A current review. Int J Oral Maxillofac Implants 2003;18(1):93-103

7. Fernández-Barbero JE, Galindo-Moreno P, Ávila-Ortiz G, Caba $\mathrm{O}$, Sánchez-Fernández E, Wang HL. Flow cytometric and morphological characterization of platelet rich plasma gel. Clin Oral Implants Res. 2006;17(6):687-93.

8. Anitua E, Andía I, Sánchez M, Azofra J, del Mar Zalduendo M, de la Fuente $M$, et al. Autologous preparations rich in growth factors promotes proliferation and induce VEGF and HGF production by human tendon cells in culture. J Orthop Res. 2005;23(2):281-6

9. Anitua E, Sánchez M, Orive G, Andía I. The potential impact of the preparation rich in growth factors (PRGF) in different medical fields. Biomaterials 2007;28(31):4551-60

10. González M, Arteaga-Vizcaíno M, Benito M, Benito M. Aplicación del plasma rico en plaquetas (PRP) y sus derivados en implantología dental y cirugía plástica. Invest Clín. [internet]. 2012 dic [citado 2016 jul 27]; 53(4):408-18

11. Kawase T, Okuda K, Wolff LF, Yoshie H. Platelet-rich plasmaderived fibrin clot formation stimulates collagen synthesis in periodontal ligament and osteoblastic cells in vitro. J Periodontol. 2003;74(6):858-64.

12. Kawase $T$, Okuda $K$, Saito $Y$, Yoshie $H$. In vitro evidence that the biological effects of platelet-rich plasma on periodontal ligament cells is not mediated solely by constituent transforminggrowth factor-beta or platelet-derived growth factor. J Periodontol. 2005;76(5):760-7.

13. Giuffre G, Caputo G, Misso S, Peluso F. Platelet-rich plasma treatment and hemostasis in patients with hemorrhagic risk. Minerva Stomatol. 2006;55(11-12):599-609.

14. Aggarwal BB. Signalling pathways of the TNF superfamily: a double-edged sword. Nat Rev Immunol. 2003;3(9):745-56.

15. Zachary I. VEGF signalling: integration and multi-tasking in endothelial cell biology. Biochem Soc Trans. 2003;31(Pt 6):1171-7.

16. Ferrara N, Gerber HP, LeCouter J. The biology of VEGF and its receptors. Nat Med. 2003;9(6):669-76.

17. Berlanga-Acosta J, Gavilondo-Cowley J, López-Saura P, González-López T, Castro-Santana MD, López-Mola E, et al. Epidermal growth factor in clinical practice - a review of its biological actions, clinical indications and safety implications. Int Wound J. 2009;6(5):331-46

18. Yates RA, Nanney LB, Gates RE, King LE Jr. Epidermal growth factor and related growth factors. Int J Dermatol. 1991;30(10):687-94

19. Agren MS, Rasmussen K, Pakkenberg B, Jorgensen B. Growth factor and proteinase profile of Vivostat $®$ platelet-rich fibrin linked to tissue repair. Vox Sang. 2014;107(1):37-43.

20. Zimmermann $R$, Jakubietz $R$, Jakubietz $M$, Strasser $E$, Schlegel A, Wiltfang J, et al. Different preparation methods to obtain platelet components as a source growth factors for local application. Transfusion 2001;41(10):1217-24.

21. Durante $C$, Agostini F, Abbruzzese L, Toffola RT, Zanolin S, Suine $C$, et al. Growth factor release from platelet concentrates: analytic quantification and characterization for clinical applications. Vox Sang. 2013;105(2):129-36.

22. Barsotti $M C$, Losi $P$, Briganti $E$, Sanguinetti $E$, Magera $A, A$ Kayal $T$, et al. Effect of platelet lysate on human cells involved in different phases of wound healing. PLoS One. 2013;8(12):e84753.

23. Molina V, Arruzazabala L, Carbajal D, Más R. Farmacología de los agentes antiagregantes plaquetarios. Revista Cenic Ciencias Biológicas. 2005;36(1):3-12

24. Patrono C, Bachmann F, Baigent C, Bode C, De Caterina R, Charbonnier B, et al. Grupo de trabajo sobre el uso de agentes antiplaquetarios en pacientes con enfermedad cardiovascular aterosclerótica de la Sociedad Europea de Cardiología. Documento de consenso de expertos sobre el uso de agentes antiplaquetarios. Rev Esp Card. 2004;57:963-80 
25. Harker LA, Boissel JP, Pilgrim AJ, Gent M. Comparative safety and tolerability of clopidogrel and aspirin: results from CAPRIE. CAPRIE Steering Committee and Investigators. Clopidogrel versus aspirin in patients at risk of ischaemic events. Drug Saf. 1999;21(4):325-35.

26. Salazar-Álvarez AE, Riera-del-Moral LF, García-Arranz M, Álvarez-García J, Concepción-Rodríguez NA, Riera-de-Cubas L. Uso de plasma rico en plaquetas para cicatrización de úlceras crónicas de miembros inferiores. Actas Dermosifiliogr. 2014;105(6):597-604.

27. Martínez-Zapata MJ, Martí-Carvajal AJ, Solà I, Expósito JA, Bolíbar I, Rodríguez L, et al. Autologous platelet-rich plasma for treating chronic wounds. Cochrane Database Syst Rev. 2016;(5):CD006899.

28. Rodríguez-Zendejas NJ, Contreras-Ruiz J, Garrido-Espíndola X, Romero-Valdovinos M, Lozano-Platonof A. Uso de plasma autólogo rico en plaquetas en úlceras de difícil cicatrización. Reporte de un caso. Med Cutan Iber Lat Am. 2014;42(1-3):62-4.

29. Tamayo M. El proceso de la investigación científica: incluye evaluación y administración de proyectos de investigación. 3a ed. México: Limusa Noriega Editores; 2005.

30. Beutler E, Lichtman M. Williams's hematology. 5th ed. New York, USA: McGraw-Hill; 1995.

31. Born GV, Cross MJ. The aggregation of the blood platelets. J Phisiol. 1963;168:178-95.

32. Anitua E. La utilización de los factores de crecimiento plasmáticos en cirugía oral, maxilofacial y periodoncia (PRGF). RCOE. 2001;6:305-15.

33. Engvall E, Perlman P. Immunosorbent assay. Immunochem. 1971;8(9):871-4.

34. Daniel WW. Bioestadística. Base para el análisis de las ciencias de la salud. 2a ed. México, D.F.: Editorial Limusa; 1991.

35. Instituto Venezolano de Investigaciones Clínicas (IVIC). Consentimiento informado. 2012 [citado 2015 jun 26]. Disponible en: www.ivic.gob.ve/bioetica

36. World Medical Association. World Medical Association Declaration of Helsinki: ethical principles for medical research involving human subjects. JAMA. 2013;310(20):2191-4.
37. Council for International Organizations of Medical Sciences Pautas éticas internacionales para la investigación biomédica en seres humanos. Ginebra: CIOMS; 2002.

38. Antoniades HN. Human platelet-derived growth factor (PDGFBB): purification of PDGFBB-I and PDGFBB-II and separation of their reduced subunits. Proc Natl Acad Sci USA. 1981;78(12):7314-7.

39. Blair P, Flaumenhaft R. Platelet alpha-granules: basic biology and clinical correlates. Blood Rev. 2009;23(4):177-89.

40. Ledent E, Wasteson A, Berlin G. Growth factor release during preparation and storage of platelet concentrates. Vox Sang 1995;68(4):205-9.

41. Reigstad $\amalg$, Varhaug JE, Lillehaug JR. Structural and functional specificities of PDGF-C and PDGF-D, the novel members of the platelet-derived growth factors family. FEBS J. 2005;272(22):5723-41.

42. Eppley BL, Woodwell JE, Higgins J. Platelet quantification and growth factor analysis from platelet-rich plasma: implications for wound healing. Plast Reconstr Surg 2004;114(6):1502-8.

43. Lee JW, Kwon OH, Kim TK, Cho YK, Choi KY, Chung HY, et al. Platelet-rich plasma: quantitative assessment of growth factor levels and comparative analysis of activated and inactivated groups. Arch Plast Surg. 2013;40(5):530-5.

44. Weibrich G, Kleis WK, Hafner G, Hitzler WE. Growth factors levels in platelet-rich plasma and correlations with donor age, sex and platelet count. J Craniomaxillofac Surg. 2002;30(2):97-102.

45. Weibrich G, Kleis WK, Hafner G. Growth factor levels in the platelet-rich plasma produced by 2 different methods: curasantype PRP kit versus PCCS PRP system. Int J Oral Maxillofac Implants 2002;17(2):184-90.

46. Zimmermann $R$, Jakubietz $R$, Jakubietz $M$, Strasser $E$, Schlegel A, Wiltfang J, et al. Different preparation methods to obtain platelet components as a source growth factors for local application. Transfusion 2001;41(10):1217-24.

47. Perseghin $P$, Sciorelli G, Belotti D, Speranza T, Pogliani Em, Ferro $\mathrm{O}$, et al. Frozen-and-thawed allogeneic platelet gels for treating postoperative chronic wounds. Transfusion. 2005;45(9):1544-6. 